\title{
Drosophila QVR/SSS Modulates the Activation and C-Type Inactivation Kinetics of Shaker $\mathrm{K}^{+}$Channels
}

\author{
Terry Dean, ${ }^{1}$ Rong Xu, ${ }^{2}$ William Joiner, ${ }^{1,3}$ Amita Sehgal, ${ }^{1,3 *}$ and Toshinori Hoshi ${ }^{2 *}$ \\ ${ }^{1}$ Department of Neuroscience, University of Pennsylvania, ${ }^{2}$ Department of Physiology, University of Pennsylvania, and ${ }^{3}$ Howard Hughes Medical Institute, \\ University of Pennsylvania, Philadelphia, Pennsylvania 19104
}

The quiver/sleepless ( $q v r / s s s)$ gene encodes a small, glycosylphosphatidylinositol-anchored protein that plays a critical role in the regulation of sleep in Drosophila. Loss-of-function mutations in qvr/sss severely suppress sleep and effect multiple changes in in situ Shaker $\mathrm{K}^{+}$currents, including decreased magnitude, slower time-to-peak, and cumulative inactivation. Recently, we demonstrated that SLEEPLESS (SSS) protein modulates Shaker channel activity, possibly through a direct interaction at the plasma membrane. We show here that SSS accelerates the activation of heterologously expressed Shaker channels with no effect on deactivation or fast N-type inactivation. Furthermore, this SSS-induced acceleration is sensitive to the pharmacological disruption of lipid rafts and sufficiently accounts for the slower time-to-peak of in situ Shaker currents seen in qvr/sss mutants. We also find that SSS decreases the rate of C-type inactivation of heterologously expressed Shaker channels, providing a potential mechanism for the cumulative inactivation phenotype induced by $q v r / s s s$ loss-of-function mutations. Kinetic modeling based on the in vitro results suggests that the SSS-dependent regulation of channel kinetics accounts for nearly $40 \%$ of the decrease in Shaker current magnitude in flies lacking SSS. Sleep duration in qvr/sss-null mutants is restored to normal by a qvr/sss transgene that fully rescues the Shaker kinetic phenotypes but only partially rescues the decrease in current magnitude. Together, these results suggest that the role of SSS in the regulation of sleep in Drosophila correlates more strongly with the effects of SSS on Shaker kinetics than current magnitude.

\section{Introduction}

Humans experience an increasing pressure to sleep when they remain awake for longer than $16 \mathrm{~h}$ (Van Dongen et al., 2003). This phenomenon, called sleep homeostasis, is a conserved hallmark of sleep (Borbély and Achermann, 1999), but its molecular mechanism is unknown. Loss-of-function mutations in the Drosophila gene quiver/sleepless ( $q v r / s s s$ ) profoundly decrease sleep and, unlike sleep-reducing alleles of other genes (e.g., Shaker, dopamine transporter), severely impair homeostatic recovery sleep following sleep deprivation (Cirelli et al., 2005; Koh et al., 2008; Wu et al., 2008). Therefore, uncovering the actions of the protein product of $q v r / s s s$, SLEEPLESS (QVR/SSS or SSS), may lend the first insight into the cellular machinery underlying sleep homeostasis.

Received Jan. 21, 2011; revised June 6, 2011; accepted June 13, 2011.

Author contributions: T.D., W.J., A.S., and T.H. designed research; T.D., R.X., and T.H. performed research; T.D., W.J., A.S., and T.H. contributed unpublished reagents/analytic tools; T.D. and T.H. analyzed data; T.D., W.J., A.S., and T.H. wrote the paper.

This work was supported by grants from the National Institutes of Health (T32HL007953 and 1F30HL099407-01 to T.D., R01GM057654 and R01GM078579 to T.H., and P01AG017628 to A.S.). A.S. is an investigator of the Howard Hughes Medical Institute. We thank Carol Ma of the Levitan laboratory at Jefferson University for demonstrating how to record from Drosophila larval muscles.

The authors declare no competing financial interests.

${ }^{*}$ A.S. and T.H. contributed equally to this work.

W. Joiner's present address: Department of Pharmacology, University of California San Diego, La Jolla, CA.

Correspondence should be addressed to either of the following: Amita Sehgal, Department of Neuroscience, University of Pennsylvania, 219 Stemmler Hall, 3450 Hamilton Walk, Philadelphia, PA 19104, E-mail: amita@mail. med.upenn.edu; or Toshinori Hoshi, Department of Physiology, University of Pennsylvania, D100 Richards Building, 3700 Hamilton Walk, Philadelphia, PA 19104, E-mail: hoshi@mail.med.upenn.edu.

DOI:10.1523/JNEUROSCI.0502-11.2011

Copyright $\odot 2011$ the authors $\quad 0270-6474 / 11 / 3111387-09 \$ 15.00 / 0$
SSS is a small ( $\sim 15 \mathrm{kDa})$, glycosylphosphatidylinositol (GPI)anchored protein predicted to contain a three-fingered Ly6 domain (Koh et al., 2008; Wu et al., 2010). Similar to the GPI-anchored, Ly6-containing protein Lynx1 that modulates acetylcholine-gated ion channels (Miwa et al., 1999), SSS appears to interact with the canonical voltage-gated $\mathrm{K}^{+}$channel Shaker, forming a stable complex when coexpressed with Shaker in vitro (Wu et al., 2010). Mutations in qvr/sss have multiple effects on Drosophila Shaker $\mathrm{K}^{+}$currents, including decreased magnitude, delayed time-to-peak, and a cumulative inactivation upon repetitive depolarization (Wang et al., 2000; Wang and Wu, 2010; Wu et al., 2010). We recently demonstrated that SSS cell autonomously modulates Shaker currents in Drosophila and that SSS coexpression alters the kinetics of heterologously expressed Shaker channels in a manner consistent with the in situ findings (Wu et al., 2010).

Several questions remain about the SSS-Shaker interaction. For example, the kinetic processes (i.e., activation, deactivation, inactivation) affected by $q v r / s s s$ are unknown. Additionally, because $q v r / s s s$ mutants express less Shaker protein (Wu et al., 2010), the contributions of the SSS-dependent kinetic effects to the net loss of Shaker current magnitude and the sleep phenotype are unclear. Finally, while lipid rafts facilitate the effects of some GPI-anchored proteins on ion channel signaling [e.g., the $\alpha_{2} \delta$ subunit of voltage-gated $\mathrm{Ca}^{2+}$ channels (A. Davies et al., 2010)], the role of rafts in the interactions between SSS and Shaker has not been explored.

We show here that SSS accelerates activation of heterologously expressed Shaker channels with little effect on deactiva- 
tion or N-type inactivation. The SSS-induced activation effect may account for the slower time-to-peak of Shaker currents in $q v r / s s s$ mutants and is sensitive to the depletion of membrane cholesterol. Coexpression with SSS also slows entry of heterologously expressed Shaker channels into the C-type inactivated state, providing a potential mechanism for the cumulative inactivation phenotype in loss-of-function qvr/sss mutants. Furthermore, kinetic modeling based on the in vitro results suggest that the effects of SSS on Shaker activation and C-type inactivation account for nearly $40 \%$ of the total loss of Shaker current magnitude in $q v r / s s s$ mutants. We propose that the regulation of sleep in Drosophila by SSS is more dependent on the modulation of Shaker current kinetics than on Shaker current magnitude.

\section{Materials and Methods}

Fly stocks and maintenance. The transgenic fly line bearing a P-element insertion in the open reading frame (exon 5) of the gene designated CG33472 (qvr/sss) by the Drosophila Genome Project has been previously described as $s s^{\mathrm{P} 1}$ (Koh et al., 2008). The sss $^{\mathrm{P} 1}$ strain was outcrossed five times to an isogenic white background stock (iso31) obtained from the Bloomington Stock Center. Individual fly lines containing the 24B-GAL4 driver and UAS-sss were generated as previously described (Wu et al., 2010) and crossed into an $s s s^{\mathrm{P} 1}$ background. Fly stocks were maintained at $25^{\circ} \mathrm{C}$ on standard molasses-yeast-cornmeal food and entrained to a 12:12 h light:dark schedule.

Cells, cell transfection, and DNA constructs. HEK-tsA cells were cultured in standard media (DMEM, 10\% FBS, $1 \%$ penicillin/streptomycin) at $37^{\circ} \mathrm{C}$ and $5 \% \mathrm{CO}_{2}$. Cells were transfected by FuGENE6 (Roche) with a plasmid DNA coding for GFP (to identify transfected cells) and equal quantities $(1.78 \mu \mathrm{g})$ of plasmid DNAs coding for SSS and $\mathrm{K}^{+}$channels in pGWI or pcDNA3 expression vectors. We used the following wild-type $\mathrm{K}^{+}$channels: Drosophila Shaker B (ShB; AAF48785), Drosophila Eag (dEag; AAA28495), and human large-conductance $\mathrm{Ca}^{2+}$-activated $\mathrm{K}^{+}$ channels (hSlo1; AAB65837). The truncated and mutant channel variants used were ShB $\Delta \mathrm{N}$ (deletion of residues 6-46) (Hoshi et al., 1990), ShB $\Delta$ N T449A, ShB $\Delta$ N W434F, and hKv1.3 H399Y (AAH35059; a gift from Dr. C. Deutsch, University of Pennsylvania, Philadelphia, PA). Substitutions in the pore region T449A and H399Y accelerate and slow the rate of C-type inactivation, respectively (López-Barneo et al., 1993), while substitution W434F results in a nonconducting ShB channel (Perozo et al., 1993).

In situ electrophysiology. For in situ recordings, muscle ionic currents were recorded from third-instar Drosophila larvae during the middle $6 \mathrm{~h}$ of the light cycle, in a manner previously described (Wu et al., 2010). Larvae were dissected at room temperature in a $\mathrm{Ca}^{2+}$-free, hemolymphlike saline [HL3.1; consisting of the following (in $\mathrm{mm}$ ): $70 \mathrm{NaCl}, 5 \mathrm{KCl}, 4$ $\mathrm{MgCl}_{2}, 10 \mathrm{NaHCO}_{3}, 5$ trehalose, 115 sucrose, 5 HEPES, pH 7.1 with $\mathrm{N}$-methyl-D-glucamine (NMG)] (Feng et al., 2004) to gain access to muscle 6 of hemisegments A3-A5. The dissection solution was replaced with $\mathrm{a} \mathrm{Ca}^{2+}$-free HL3.1 recording solution containing 5 mM EGTA and either $5 \mathrm{~mm}$ (physiological) or $30 \mathrm{~mm}$ (supraphysiological) $\mathrm{KCl}$. The two ends of the target muscle were impaled with a voltage-sensing electrode and a current-injecting electrode, both coated with Sylgard (Dow Corning). When filled with $2 \mathrm{M} \mathrm{KCl}$ (with $10 \mathrm{~mm}$ EGTA, pH 7.2 with $\mathrm{KOH}$ ), the electrodes had initial resistances of $8-20$ and $4-10 \mathrm{M} \Omega$, respectively. Signals were amplified (OC-725C; Warner), low-pass filtered at $1 \mathrm{kHz}$ (8-pole Bessel, model 902; Frequency Devices), digitized using an $\mathrm{AD} / \mathrm{DA}$ converter (ITC16; HEKA), and recorded/stored via custom data acquisition software. Igor Pro 6.1 (WaveMetrics) was used for post hoc analysis of the data.

Muscles were voltage-clamped at $-80 \mathrm{mV}$ and depolarized (e.g., to 10 $\mathrm{mV}$ ) either directly from holding potential to elicit $I_{\mathrm{A}}$ and $I_{\mathrm{K}} \mathrm{K}^{+}$currents or after a $200 \mathrm{~ms}$ prepulse to $-20 \mathrm{mV}$ to elicit $I_{\mathrm{K}}$ alone. Subtraction of the two series of sweeps yielded $I_{\mathrm{A}}$ alone. Unless specified, pulses were applied every $\sim 2 \mathrm{~s}$.

Ionic currents were normalized by cell capacitance, calculated from currents recorded during a voltage ramp by the following relationship:
$I_{\text {ramp }}-C(\mathrm{dV} / \mathrm{dt})=I_{\text {step }}$, where $I_{\text {ramp }}$ is recorded at a particular voltage during a ramp of ${ }^{\mathrm{dV}} / \mathrm{dt}$, and $I_{\text {step }}$ is recorded at steady state at the same voltage (Wu et al., 2010).

Macroscopic capacitive and leak currents were subtracted using a $P / 4$ protocol. All data are presented as mean \pm SEM. The statistical significance of differences in electrophysiology metrics was determined by twotailed Student's $t$ tests. Comparisons involving multiple groups were analyzed by two-way ANOVA, followed by Student's $t$ tests corrected with the Bonferroni method. Statistical significance was assumed at $p \leq 0.05$.

In vitro electrophysiology. Inside-out and whole-cell patch-clamp recordings were conducted at room temperature $\left(21-23^{\circ} \mathrm{C}\right) 2 \mathrm{~d}$ after transfection. The extracellular recording solution contained the following (in $\mathrm{mm}$ ): $130 \mathrm{NaCl}, 10 \mathrm{KCl}, 2 \mathrm{CaCl}_{2}, 2 \mathrm{MgCl}_{2}, 10 \mathrm{HEPES}$, and 15 glucose ( $\mathrm{pH}$ 7.4 with NMG). The intracellular solution contained the following (in mM): $140 \mathrm{KCl}, 2 \mathrm{MgCl}_{2}, 11$ EGTA, and 10 HEPES (pH 7.2 with NMG). Output signals from the patch-clamp amplifier (Axopatch 200A; Molecular Devices) were filtered at $10 \mathrm{kHz}$ and digitized at $100 \mathrm{kHz}$. Data were recorded, stored, and analyzed as described above. Ionic and gating currents were elicited by depolarizing pulses from a holding voltage of -100 $\mathrm{mV}$. Unless specified, the typical interpulse interval was $\sim 3 \mathrm{~s}$. Macroscopic capacitive and leak currents were subtracted using a $P / 8$ protocol. Data were analyzed as above for in situ electrophysiology.

Modeling of Shaker currents. Zagotta et al. (1994a) proposed a model of Shaker gating based on experimental observations of ShB expressed in Xenopus oocytes. An abbreviated form of the $4 \times 2$-step model is outlined in Figure $3 A$ and further described in Results, below. The original rate constant values were as follows (in s ${ }^{-1}$ ): $\alpha=1120, \beta=373, \gamma=$ 2800 , and $\delta=21.2$. The rate constants for transitions after channel opening (not depicted in the abbreviated form) were $O \rightarrow C_{\mathrm{f}}=600$ and $C_{\mathrm{f}} \rightarrow \mathrm{O}=3800$. Adjusting these parameters to fit our experimental observations only required adjusting $\alpha$ to 616 . Further manipulations of the forward rate constants $\alpha$ and $\gamma$ are described in the Results. The voltage dependence of the rate constants was not altered.

Drosophila sleep assay. Adult female Drosophila (3- to 5-d-old) were individually loaded into glass locomotor tubes compatible with the Drosophila Activity Monitoring System (Trikinetics); one end of each tube was filled with yarn while the other contained a 5\%:2\% sucrose:agar solution with or without additional $\mathrm{KCl}$ supplementation. After $1 \mathrm{~d}$ of acclimation, locomotor activity was recorded over 4 consecutive days, processed via DAMFileScan (Trikinetics), and analyzed via pySolo sleep analysis software (Gilestro and Cirelli, 2009). As has been established, sleep was defined as periods of inactivity lasting at least $5 \mathrm{~min}$ (Pfeiffenberger et al., 2010).

Sodium bicarbonate extraction of lipid rafts. HEK-tsA cells in $100 \mathrm{~mm}$ culture dishes were transfected as described above. Culture medium was replaced with the extracellular recording solution described above and allowed to stand at room temperature. After $30 \mathrm{~min}$, cells were washed twice with ice-cold PBS and homogenized over ice in $2.1 \mathrm{ml}$ of $500 \mathrm{~mm}$ $\mathrm{NaHCO}_{3}$ (pH 11 with $\mathrm{NaOH}$ ). The homogenate was then sonicated by six $5 \mathrm{~s}$ pulses with a wand sonicator (Model 100; Fisher). Two milliliters of the homogenate were mixed with an equal volume of $90 \%$ sucrose (w/v) in MES-buffered saline (MBS; Boston Bioproducts) and placed in an UltraClear ultracentrifuge tube (Beckman Coulter). The tube was then layered with $4 \mathrm{ml}$ of $30 \%$ sucrose in MBS, $250 \mathrm{~mm} \mathrm{NaHCO}_{3}, \sim 5 \mathrm{ml}$ of $5 \%$ sucrose in MBS, and $250 \mathrm{~mm} \mathrm{NaHCO}_{3}$. The tube was centrifuged at $>175,000 \mathrm{~g}$ (sw40-Ti rotor, L8-M UC; Beckman Coulter) for $20-23 \mathrm{~h}$ at $4^{\circ} \mathrm{C}$. Serial fractions of $0.5-1 \mathrm{ml}$ were harvested from the top of the sucrose gradient and frozen at $-80^{\circ} \mathrm{C}$ for later analysis by Western blotting.

Western blot analysis. Sucrose gradient aliquots were thawed, supplemented with SDS sample buffer, and boiled for $5 \mathrm{~min}$ before being loaded onto a $4-15 \%$ Tris-HCl SDS-PAGE gel (Bio-Rad). Identical volumes were loaded in each lane. The proteins were transferred to nitrocellulose membranes (Bio-Rad), which were detected by primary antibodies to Sh (1:1000; UPGP57), SSS (1:1000; UPGP69), caveolin (1:2000; polyconal \#610060, BD Transduction Laboratories), and $\beta$-adaptin (1:2000; \#610382, BD Transduction Laboratories) and secondary antibodies at 1:2000-3000. Membranes were stripped with Western blot stripping 
buffer (Thermo Scientific) before exposure to a second primary antibody. Immunoreactivity was visualized by chemiluminescence reagents (ECLPlus; GE Life Sciences) and exposure to X-ray film. X-ray film was scanned into Photoshop where only rudimentary manipulations (i.e., brightness and contrast) were applied to the entire blot.

\section{Results}

SSS specifically affects the activation kinetics of Shaker $\mathrm{K}^{+}$channels

Shaker channels are the sole source of the fast-activating, fastinactivating $\mathrm{K}^{+}$current (A-type current, $I_{\mathrm{A}}$ ) in Drosophila larval muscles (Wu and Haugland, 1985). As previously reported (Wu et al., 2010), larvae bearing mutations in qvr/sss exhibit substantially decreased $I_{\mathrm{A}}$ magnitude (control: $23.84 \pm 3.15 \mathrm{nA} / \mathrm{nC}$; sss $\left.^{\mathrm{P} 1}: 3.87 \pm 0.44 \mathrm{nA} / \mathrm{nC} ; p<0.001\right)$ (Fig. $1 \mathrm{~A}$ ) and a slower $I_{\mathrm{A}}$ time-to-peak (control: $2.60 \pm 0.10 \mathrm{~ms}$; sss $^{\mathrm{P} 1}$ : $4.08 \pm 0.17 \mathrm{~ms} ; p<$ 0.001 ), while in vitro SSS coexpression significantly decreases wild-type ShB time-to-peak (ShB: $6.71 \pm 0.57 \mathrm{~ms}$; ShB + SSS: $4.58 \pm 0.38 \mathrm{~ms} ; p<0.001$ ) (Fig. $1 B$ ). Concomitant with time-topeak, SSS also significantly accelerated the rate of ShB current decay (Fig. $1 B, F$ ) at certain voltages, but did not affect the rate of recovery from decay (Fig. 1G). The lack of an effect of SSS coexpression on the activation time courses of wild-type dEAG, wildtype hSlo1, or hKv1.3 H499Y currents (Fig. 1C-E) demonstrated the preferred action of SSS on Shaker channels.

The faster time-to-peak and decay of ShB currents in the presence of SSS could be caused by changes in the activation, deactivation, and/or inactivation kinetics of the ShB channel. Although SSS coexpression accelerated the rate of ShB current decay at voltages $\leq 30 \mathrm{mV}$, the effect of SSS diminished at increasingly depolarized voltages, failing to reach statistical significance at voltages $>30 \mathrm{mV}$ (Fig. $1 \mathrm{~F}$ ). This observed convergence in current decay rates at depolarized voltages is consistent with the possibility that SSS primarily affects the rate of ShB activation. Activation and N-type inactivation of the Shaker channel are tightly coupled, and the rate constant of entry into the inactivated state is largely independent of voltage (Zagotta et al., 1989; Hoshi et al., 1990). Thus, at relatively hyperpolarized voltages at which the kinetics of activation and inactivation are comparable, activation kinetics often influence the time course of current decay. However, at depolarized voltages, where activation occurs much faster than inactivation, the rate of current decay is less affected.

To assess the effect of SSS on ShB channel kinetics in the absence of inactivation, we used the $\operatorname{ShB} \Delta \mathrm{N}$ channel, a well characterized deletion mutant in which $\mathrm{N}$-type inactivation is disrupted (Hoshi et al., 1990). SSS coexpression significantly decreased the time-to-half-maximum of $\mathrm{ShB} \Delta \mathrm{N}$ currents $(\mathrm{ShB} \Delta \mathrm{N}: 8.88 \pm 0.67 \mathrm{~ms} ; \mathrm{ShB} \Delta \mathrm{N}+$ SSS: $5.38 \pm 0.38 \mathrm{~ms} ; p<$ 0.001 ) (Fig. $2 A$ ) and the time constant of activation was significantly smaller throughout the entire voltage range from -30 to $75 \mathrm{mV}$ (Fig. 2 B). For example, at $75 \mathrm{mV}$, where the opening rate constants dominate and the closing rate constant values are negligible, SSS decreased the activation time constant by $\sim 22 \%$. The voltage dependence of the activation time constant was not altered by SSS. Furthermore, despite the significant effect of SSS on the $\operatorname{ShB} \Delta \mathrm{N}$ current activation, the rate of deactivation remained unaffected (Fig. 2C). In summary, these data are consistent with a SSS-induced acceleration of Sh activation kinetics, with little to no effect on inactivation or deactivation.

Analysis of the conductance-voltage (GV) relationships from $\mathrm{ShB} \Delta \mathrm{N} \pm$ SSS traces showed that SSS shifts the half-activation voltage $\left(\mathrm{V}_{0.5}\right)$ of $\mathrm{GV}$ toward the negative direction (i.e., a leftshift) by $\sim 10 \mathrm{mV}(\mathrm{ShB} \Delta \mathrm{N}:-37.0 \pm 2.8 \mathrm{mV}$; ShB $\Delta \mathrm{N}+\mathrm{SSS}$ :
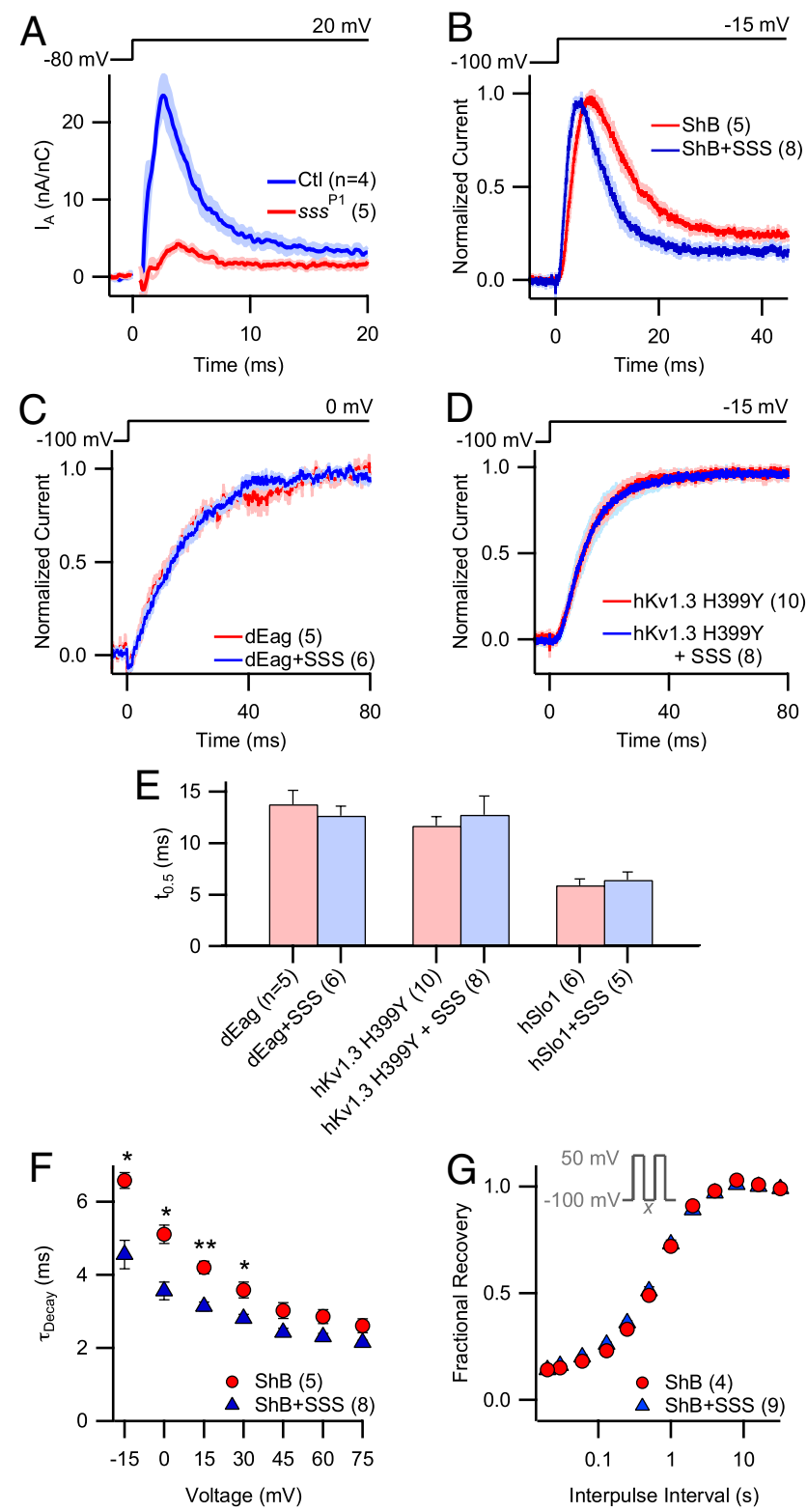

Figure 1. SSS produces similar kinetic effects in vitro and in vivo.A, Pooled $I_{A}$ traces (mean \pm $\mathrm{SEM}$ ) collected by two-electrode voltage-clamp recordings from sss ${ }^{\mathrm{P} 1}$ mutant and control (Ctl) Drosophila larval muscles at $20 \mathrm{mV}$. The capacitative transients were removed for clarity. $\boldsymbol{B}-\boldsymbol{D}$ Pooled current traces (mean \pm SEM) collected by patch-clamp recordings from HEK cells heterologously expressing $\mathrm{K}^{+}$channels with and without SSS. B, ShB currents were recorded from inside-out patches at $-15 \mathrm{mV}$. C, Whole-cell dEag currents were recorded at $0 \mathrm{mV}$. D, hKv1.3 $\mathrm{H} 399 \mathrm{Y}$ currents were recorded from inside-out patches at $-15 \mathrm{mV}$. $\boldsymbol{E}$, Time-to-half-maximum $\left(t_{0.5}\right)$ of dEag, hKv1.3, and hSlo1 currents at $0,-15$, and $100 \mathrm{mV}$, respectively, with and without SSS coexpression. $\boldsymbol{F}$, Rate of decay of ShB current ( $\left.\tau_{\text {Decay }}\right)$ with and without SSS coexpression at voltages between -15 and $75 \mathrm{mV}$. $\tau_{\text {Decay }}$ was measured by fitting a single exponential curve to the falling phase of ShB current traces at the indicated voltage. Comparisons of $\tau_{\text {Decay }}$ were only significantly different at voltages $\leq 30 \mathrm{mV}$. G, Rate of recovery of ShB from inactivation. Frac tional recovery of $S h B$ current observed during the second test pulse as a function of interpulse interval duration ( $x$ in the Inset). Inset, Standard two-pulse protocol in which patches were delivered two $50 \mathrm{~ms}$ test pulses to $50 \mathrm{mV}$ separated by a variable interpulse interval (ranging from 0.16 to $32 \mathrm{~s}$ ) at $-100 \mathrm{mV}$. ${ }^{*} p<0.05,{ }^{* *} p<0.01$.

$-46.7 \pm 1.1 \mathrm{mV}, p<0.001$ ) (Fig. $2 D$ ). Single-channel currentvoltage relationship (iV) was not affected (data not shown).

The kinetic effect of SSS on ShB $\Delta \mathrm{N}$ is cholesterol-dependent SSS is a GPI-anchored protein, and many GPI-anchored proteins are thought to be associated with cholesterol-rich membrane do- 

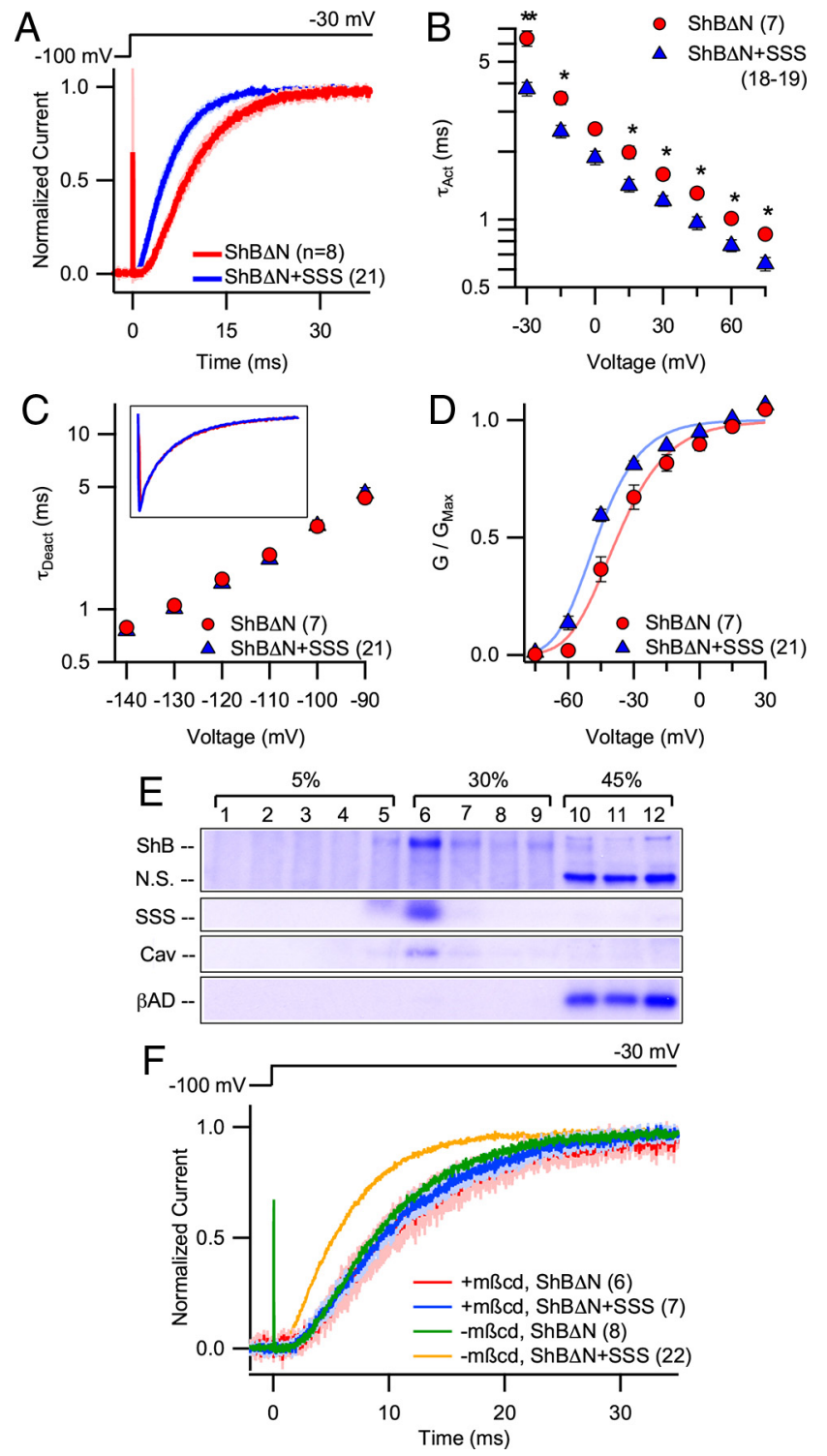

Figure 2. SSS coexpression accelerates activation of ShB $\Delta \mathrm{N}$ in a cholesterol-dependent manner. $A$, Pooled current traces (mean \pm SEM) at $-30 \mathrm{mV}$ collected by inside-out patch-clamp recordings from HEK cells heterologously expressing ShB $\Delta N$ with and without SSS. $B, \operatorname{ShB} \Delta N$ activation rate $\left(\tau_{\text {Act }}\right)$ with and without SSS coexpression measured at voltages between -30 and $75 \mathrm{mV}$. $\tau_{\text {Act }}$ was measured by fitting a single exponential curve to the second half of the rising phase (i.e., from halfmaximum to maximum amplitude) of $\operatorname{ShB} \Delta \mathrm{N}$ current traces. $\mathrm{C}, \mathrm{ShB} \Delta \mathrm{N}$ deactivation rate $\left(\tau_{\text {Deact }}\right)$ with and without SSS coexpression measured at voltages between -140 and $-90 \mathrm{mV}$. $\tau_{\text {Deact }}$ was measured by fitting a single exponential curve to the decay of $\mathrm{ShB} \Delta \mathrm{N}$ current trace from a test pulse of $45 \mathrm{mV}$ to the indicated voltage. Inset, Pooled ShB $\Delta N$ deactivation current trace (mean) at $-130 \mathrm{mV}$ $\left(n_{\text {ShB } \Delta \mathrm{N}}=8 ; n_{\text {ShB } \Delta \mathrm{N}}+\right.$ SSS $\left.=21\right) \cdot \boldsymbol{D}, \mathrm{GV}$ curves for ShB $\Delta \mathrm{N}$ with and without SSS coexpression. $\mathrm{V}_{0.5}$ was calculated after fitting a Boltzmann function raised to the fourth power to $\mathrm{GV}$ curves generated from tail currents for each patch. $\boldsymbol{E}$, Western blot of proteins from HEK cells transfected with ShB and SSS. Cells were homogenized in a $500 \mathrm{~mm} \mathrm{NaHCO}_{3}$ (pH 11) solution and segregated via sucrose gradient centrifugation. A single nitrocellulose membrane was probed for each of the following proteins: ShB, SSS, caveolin (Cav), and $\beta$-adaptin ( $\beta$ AD). Nonspecific bands (N.S.) also appear upon labeling with Shaker antibody. $\boldsymbol{F}$, Pooled current traces (mean \pm SEM) at $-30 \mathrm{mV}$ collected by inside-out patch-clamp recordings of $S h B \Delta N$ with and without $S S S$ from HEK cells preincubated for $30 \mathrm{~min}$ in 5 $\mathrm{mm} \mathrm{m} \beta c \mathrm{~d}$ (blue \pm light blue and red \pm pink, respectively). Mean traces without $\mathrm{m} \beta c \mathrm{c}$ preincubation (as in Fig. 2 A) are provided for comparison (green and orange). ${ }^{*} p<0.05,{ }^{* *} p<0.01$.

mains in Drosophila (Rietveld et al., 1999). These domains, called lipid rafts, are implicated in the modulation of voltage-gated $\mathrm{K}^{+}$ channel activity (Martens et al., 2001), leading us to hypothesize a role for lipid rafts in the interaction between SSS and Shaker channels. To determine whether SSS and/or ShB can traffic to lipid rafts, we performed a detergent-free isolation of lipid rafts from HEK cells heterologously expressing ShB and SSS. Like caveolin, a known component of lipid rafts, both ShB and SSS localized preferentially to low-density, lipid raft-containing membrane fractions, while $\beta$-adaptin, a protein typically excluded from lipid rafts, did not (Fig. 2E).

We also investigated the importance of membrane cholesterol for the effect of SSS on Shaker by using methyl- $\beta$-cyclodextrin $(\mathrm{m} \beta \mathrm{cd})$, a cholesterol-chelating agent (Yancey et al., 1996). Incubation with $\mathrm{m} \beta \mathrm{cd}(5 \mathrm{~mm})$ for $30 \mathrm{~min}$ had minimal effects on $\mathrm{ShB} \Delta \mathrm{N}$ activation kinetics at $-30 \mathrm{mV}$; however, it was sufficient to completely ablate the SSS-induced acceleration of ShB $\Delta \mathrm{N}$ activation (Fig. 2 F).

\section{Qualitative modeling of $S h B \Delta N$ activation predicts SSS-induced changes in the GV relationship and gating charge movement}

Measurements of single-channel currents and macroscopic ionic and gating currents of ShB channels led Zagotta and colleagues (Hoshi et al., 1994; Zagotta et al., 1994a,b) to propose a model of Shaker activation in which opening of the ion conduction gate is preceded by two coupled transitions in each of the four independent subunits. An abbreviated form of this model (the ZHA model) is depicted in Figure $3 A$. While a recent study suggests that each subunit may undergo four transitions before the main gate opens (Tao et al., 2010), the ZHA model adequately describes most aspects of ShB channel gating in an experimentally constrained manner. Similarly, with only minor modifications of the rate constant values originally derived from data collected from Xenopus oocytes, the ZHA model described ShB $\Delta \mathrm{N}$ ionic currents measured from patches from HEK-tsA cells (see Materials and Methods, above). Thus, we sought to identify the minimal change in the ZHA model required to account for the effects of SSS on ShB $\Delta \mathrm{N}$ kinetics.

To proceed, we first determined whether SSS affected the sigmoidicity of ShB $\Delta \mathrm{N}$ current activation. Introduced by Zagotta et al. (1994b), sigmoidicity of a current trace quantifies the relative amount and distribution of delay in ion channel opening. Thus, sigmoidicity comparisons may detect alterations in the number of conformational changes that a channel undergoes before it opens or indicate unequal changes in the kinetics of individual subunits. Recordings from patches of $\operatorname{ShB} \Delta \mathrm{N}$ with and without SSS coexpression exhibited very similar sigmoidicities (Fig. 3B), consistent with the idea that ShB, with or without SSS, undergoes the same number of transitions and that all four subunits are equally affected by SSS. The sigmoidicity comparison and aforementioned observation that coexpression with SSS affects activation kinetics without affecting the voltage dependence of activation time constant (Fig. $2 B$ ) prompted us to increase the value of the forward rate constant $\alpha$ in the ZHA model (Fig. 3A) without altering its partial charge movement. Deactivation was not affected by SSS (Fig. 2C) and consequently the backwards rate constants were unaltered. Because we noticed considerable patch-to-patch variability in the speed of Shaker current activation upon SSS coexpression, we selected three sample traces (Fig. $3 C$, black) that covered the continuous range of speeds that we observed: slow (from the $0-25$ th percentile), moderate (from the 40-60th percentile), and fast (from the 75-100th percentile). While no increase in $\alpha$ was necessary to fit our ZHA baseline trace with the slow ShB $\Delta \mathrm{N}+$ SSS trace, increasing $\alpha$ by $55 \%$ (Fig. $3 C$, blue) and $136 \%$ (Fig. 3C, green) was necessary to match the moderate and fast $\operatorname{ShB} \Delta \mathrm{N}+$ SSS traces. Increasing $\alpha$ in the ZHA 
A
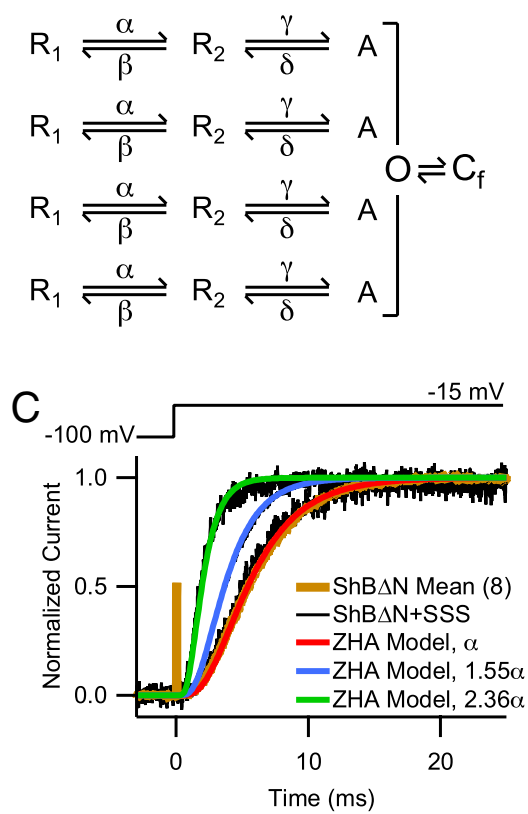

E
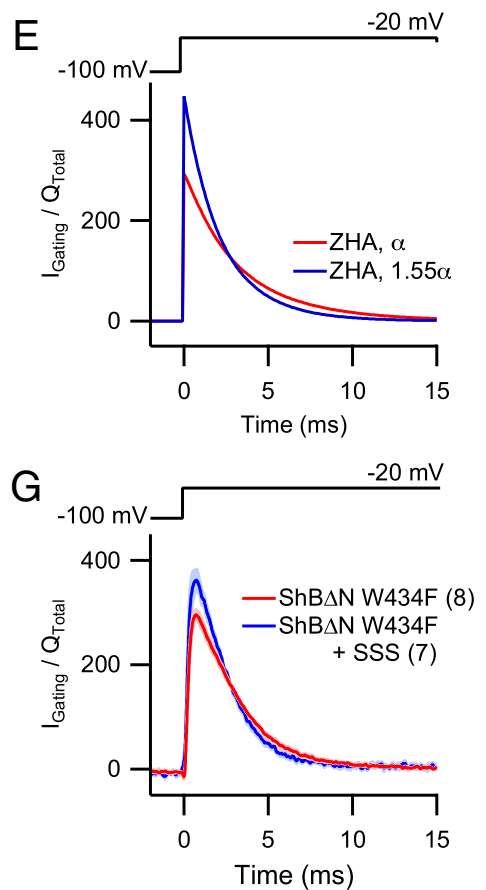

B
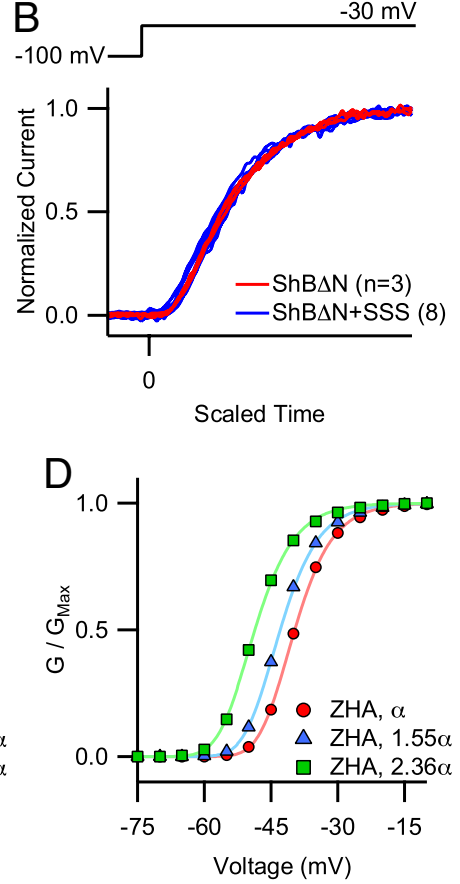

$\mathrm{F}$
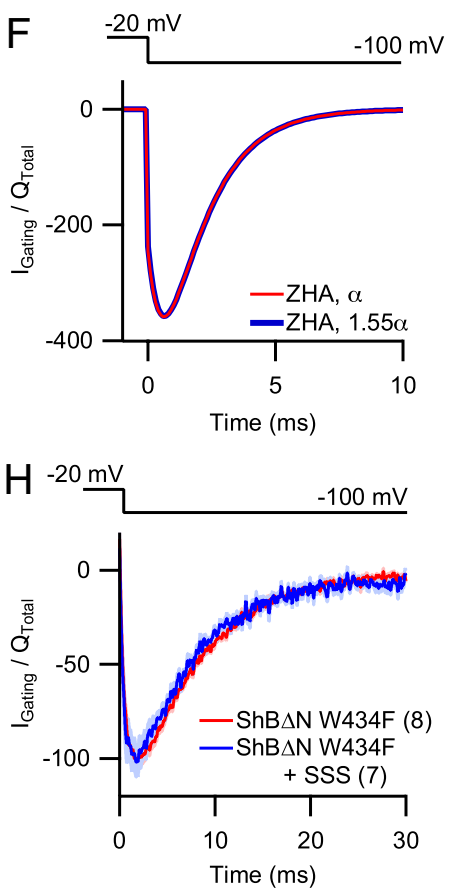

Figure 3. Increasing the activation rate in kinetic models of Shaker accurately predicts GV and gating current effects. $\boldsymbol{A}$, Abbreviated form of the $4 \times 2$-step model (see Class D in Zagotta et al., 1994a) depicting two voltage-dependent conformational changes per subunit before a final, concerted transition. $\alpha$ and $\gamma$ are forward rate constants (i.e., rate constants of activation) and $\delta$ and $\beta$ are backward rate constants (i.e., rate constants of deactivation). $\boldsymbol{B}$, Sigmoidicity comparison of individual traces of $\mathrm{ShB} \Delta \mathrm{N}$ with and without SSS coexpression. As described by Zagotta et al. (1994b), 11 individual current traces were scaled by normalizing the vertical and horizontal axes so that the maximum current and slope at half-maximum current were the same for each. $C$, Individual traces depicting patch-clamp recordings of ShB $\Delta \mathrm{N}$ current with and without SSS (black and brown, respectively) overlayed with simulated traces generated from the ZHA two-transition kinetic model of Shaker (red, blue, green). A baseline ZHA trace (red) was established by fitting the model to the pooled ShB $\Delta \mathrm{N}$ trace (brown). No change in forward rate constant $\alpha$ was required to fit the baseline ZHA trace to the slow ShB $\Delta \mathrm{N}+$ SSS trace (slowest black trace). Multiplying $\alpha$ by 1.55 and 2.36 (blue and green, respectively) was needed to match the moderate and fast ShB $\Delta N+$ SSS traces, respectively. D, GV relationship for ZHA modeled channels based on a previously established rate constant for $\alpha$ compared with values of $1.55 \alpha$ and $2.36 \alpha . \boldsymbol{E}, \boldsymbol{F}, \mathrm{ON}(\boldsymbol{E})$ and OFF $(\boldsymbol{F})$ gating current traces normalized by total charge movement generated by the ZHA model at baseline and $1.55 \alpha . G$, Normalized gating current from pooled whole-cell recordings (mean \pm SEM) of ShB $\Delta N$ W434F with and without SSS coexpression upon depolarization to $-20 \mathrm{mV}$. $\boldsymbol{H}$, Normalized current from pooled whole-cell recordings (mean \pm SEM) of $\mathrm{ShB} \Delta \mathrm{N}$ W434F with and without SSS coexpression upon repolarization to $-100 \mathrm{mV}$.

model by $55 \%$ and $136 \%$ also left-shifted the $\mathrm{GV} \mathrm{V}_{0.5}$ values from $-39.7 \mathrm{mV}$ to $-42.9 \mathrm{mV}$ and $-48.6 \mathrm{mV}$, respectively (Fig. 3D). These predicted voltage shifts are similar to the experimentally observed left shift in the $\mathrm{V}_{0.5}$ of the ShB + SSS group (Fig. 2D).

The ZHA model also predicted that increasing $\alpha$ would affect the time course of gating charge movement upon depolarization. The $55 \%$ increase in $\alpha$ required to simulate moderate ShB $\Delta \mathrm{N}+$ SSS activation kinetics was predicted to accelerate the ON gating current (Fig. 3E) with minimal effect on the OFF gating current (Fig. $3 F)$. Gating currents measured from whole cells expressing $\mathrm{ShB} \Delta \mathrm{N}$ W434F showed that SSS coexpression accelerated ON gating current kinetics in a manner similar to the ZHA predictions without appreciably affecting the OFF gating current (Fig. 3G,H). The ZHA model also predicted an approximate $4 \mathrm{mV}$ left-shift in the Q/V curve; however, we were unable to resolve such a difference and suspect that the small change was below our experimental detection limit (data not shown).

As described above, the effects of SSS on ShB activation kinetics are well accounted for by increasing $\alpha$, one of the two forward rate constants of the ZHA model (Fig. $3 A$ ). In contrast, simulations in which we instead increased the other forward rate constant $\gamma$ did not readily produce currents consistent with the experimental observations. Specifically, the increases in $\gamma$ necessary to match the SSSinduced acceleration of activation at -30 $\mathrm{mV}$ do not continue to produce appreciably faster currents at very depolarized voltages (e.g., $60 \mathrm{mV}$ ) (data not shown), which is inconsistent with experimental observations (Fig. 2B). Furthermore, increasing $\gamma$ produces a noticeable rising phase in ON gating current, which was absent from our gating current recordings (Fig. $3 G$ ). While we cannot exclude the possibility that SSS affects both $\alpha$ and $\gamma$, it is clear that $\alpha$ manipulations alone are sufficient to reproduce the SSS effects that we have observed.

\section{SSS confers resistance to C-type inactivation in vitro and in situ} Shaker channels show two distinct inactivation processes, $\mathrm{N}$-type and C-type inactivation (Hoshi et al., 1991). We evaluated whether SSS affects the rate of C-type inactivation in the ShB channel. C-type inactivation in the wild-type ShB channel is slow, occurring on the order of many seconds and masked by faster $\mathrm{N}$-type inacti- 

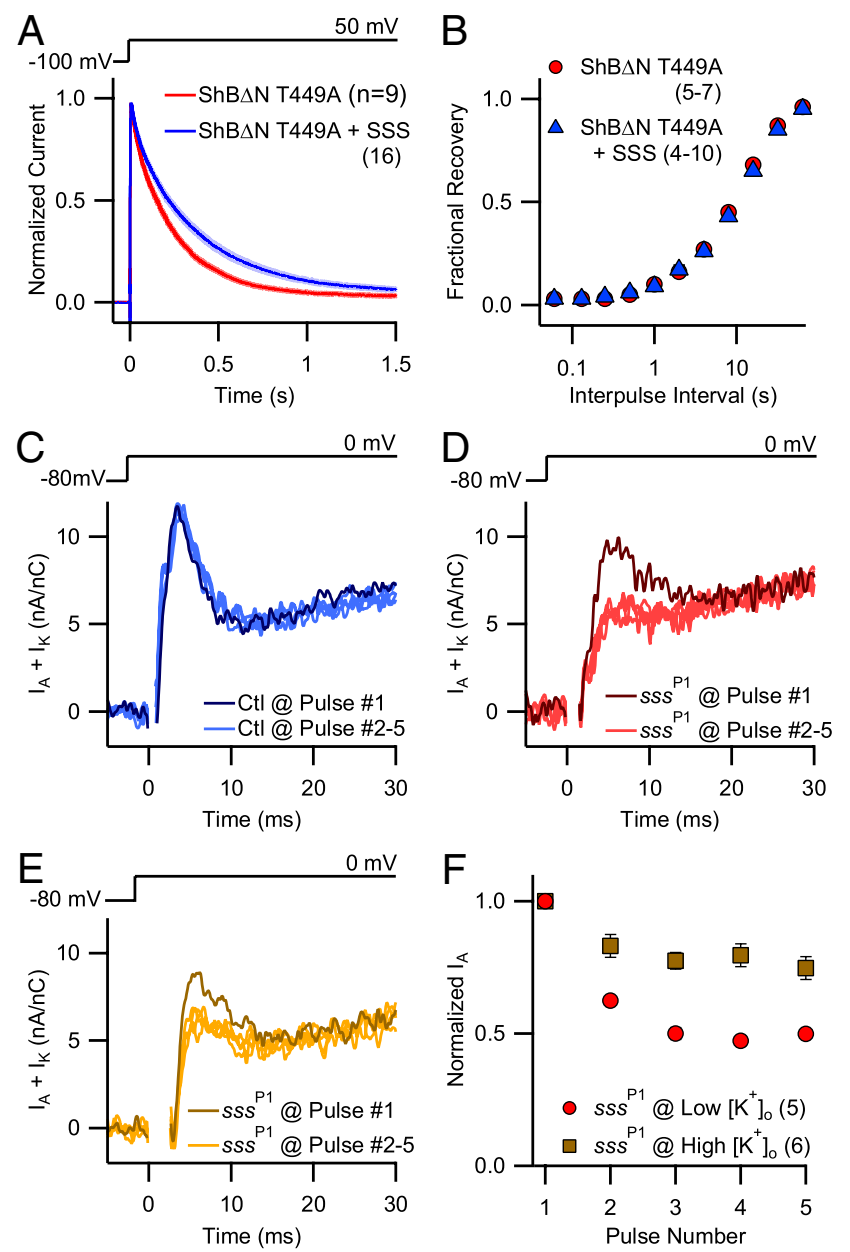

Figure 4. An SSS effect on Shaker C-type inactivation can explain the emergence of cumulative inactivation of $I_{\mathrm{A}}$ in sss mutants. $\boldsymbol{A}$, Pooled current traces (mean \pm SEM) collected by inside-out patch-clamp recordings from HEK cells heterologously expressing $\operatorname{ShB} \Delta \mathrm{N} T 449 \mathrm{~A}$ with and without SSS. B, Fractional recovery of ShB $\Delta N$ T449A channels as a function of time between two $50 \mathrm{~ms}$ test pulses at $50 \mathrm{mV}$. Channels were allowed to recover at $-100 \mathrm{mV}$ with interpulse intervals ranging between 0.06 and $64 \mathrm{~s}$. $\boldsymbol{C}-\boldsymbol{E}$, Representative serial $\mathrm{K}^{+}$current traces collected by two-electrode voltage-clamp recordings from larval Drosophila muscle at physiological $(\boldsymbol{C}, \boldsymbol{D})$ and supraphysiological $(\boldsymbol{E})\left[\mathrm{K}^{+}\right]_{0}(5 \mathrm{vs} 30 \mathrm{~mm}) . \mathrm{K}^{+}$currents were elicited from control $(C \mathrm{Cl})(\boldsymbol{C})$ and $s s^{\mathrm{P} 1}$ mutant animals $(\boldsymbol{D}, \boldsymbol{E})$ by repeated depolarization to $0 \mathrm{mV}$ every 4 s from a resting potential of $-80 \mathrm{mV}$. The Shaker-dependent component of $\mathrm{K}^{+}$current $\left(I_{\mathrm{A}}\right)$ is the fast-activating, fast-inactivating component in the depicted traces. Traces from the first five consecutive pulses (Pulse \#1 in darker colors than Pulses \#2-5) are superimposed to visualize changes in current magnitude. Capacitative transients were deleted to improve clarity. $\boldsymbol{F}$, Normalized peak $I_{A}$ magnitude upon repeated depolarization in physiological $(5 \mathrm{~mm})$ and supraphysiological $(30 \mathrm{~mm})\left[\mathrm{K}^{+}\right]_{0}$. Data were collected as in $\boldsymbol{C}-\boldsymbol{E}^{*}{ }^{*} p<0.05,{ }^{* *} p<0.01$.

vation (Hoshi et al., 1991), making it difficult to study experimentally. In the mutant $\mathrm{ShB} \Delta \mathrm{N}$ T449A, C-type inactivation occurs in a more experimentally manageable range (LópezBarneo et al., 1993). Coexpression with SSS significantly slowed the time course of C-type inactivation in the ShB $\Delta \mathrm{N}$ T449A channel $\left(\tau_{\text {inact, }}=0.393 \pm 0.027\right.$ vs $\left.0.245 \pm 0.008 \mathrm{~s}, p>0.001\right)$ (Fig. $4 A$ ), but did not affect the time course of recovery from C-type inactivation (Fig. $4 \mathrm{~B}$ ).

Earlier reports noted that $q v r / s s s$ mutant flies exhibit cumulative inactivation of $I_{\mathrm{A}}$ upon repetitive depolarization that is not observed in wild-type animals (Wang et al., 2000; Wang and Wu, 2010). The cumulatively inactivated component was named $I_{\mathrm{AS}}$, but the mechanism of loss is unknown. Because we did not observe any changes in the rate of recovery from $\mathrm{N}$-type inactivation in ShB expressed in HEK cells (Fig. 1G), we hypothesize that Shaker channels in the absence of SSS, as in Drosophila sss ${ }^{\mathrm{P} 1}$ mutants, more readily proceed to a C-type inactivated state.

Measuring the rate of C-type inactivation in Drosophila larval muscles is technically challenging, as the current size following $\mathrm{N}$-type inactivation is miniscule at best. We looked instead for a key characteristic of C-type inactivation: sensitivity to extracellular $\mathrm{K}^{+}$. Previous studies have demonstrated that elevating extracellular $\left[\mathrm{K}^{+}\right]\left(\left[\mathrm{K}^{+}\right]_{\mathrm{o}}\right)$ slows the time course of C-type inactivation in Shaker channels (Choi et al., 1991; López-Barneo et al., 1993) and we thus measured the amplitude of $I_{\mathrm{A}}$ upon repetitive depolarization at different concentrations of $\left[\mathrm{K}^{+}\right]_{\mathrm{o}}$. At a physiological level of $\left[\mathrm{K}^{+}\right]_{\mathrm{o}}(5 \mathrm{mM})$, the $I_{\mathrm{A}}$ component in wildtype flies recovered from inactivation completely between the depolarizing pulses so that the peak current magnitude was stable with repeated stimulation (Fig. 4C). Similar results were found at supraphysiological (30 mM) $\left[\mathrm{K}^{+}\right]_{\mathrm{o}}$ (data not shown). In contrast, the $I_{\mathrm{A}}$ component in sss $^{\mathrm{P} 1}$ flies dramatically decreased after the first depolarization pulse (Fig. $4 D, F$ ), in a manner previously described for a $q v r / s s s$ hypomorph (Wang and $\mathrm{Wu}, 2010$ ). As expected for the antagonistic effect of high extracellular $\mathrm{K}^{+}$on C-type inactivation, $I_{\mathrm{A}}$ in sss ${ }^{\mathrm{P} 1}$ flies was more resistant to cumulative inactivation when $\left[\mathrm{K}^{+}\right]_{\mathrm{o}}$ was increased to $30 \mathrm{mM}(51.1 \pm 1.3 \%$ vs $25.2 \pm 4.3 \%, p<0.001$ ) (Fig. $4 E, F$ ). Because extracellular $\mathrm{K}^{+}$ions could decrease fast inactivation by removing the inactivation particle from its binding pocket (Demo and Yellen, 1991), we also measured the rate of $I_{\mathrm{A}}$ decay at 5 and $30 \mathrm{~mm}\left[\mathrm{~K}^{+}\right]_{\mathrm{o}}$, but found no significant difference $\left(\tau_{\text {Decay }}=2.6 \pm 0.1\right.$ vs $2.6 \pm 0.2 \mathrm{~ms}$, respectively). Separately, expression of SSS in muscles of $s s s^{\mathrm{P} 1}$ mutants significantly decreased the proportion of cumulatively inactivated current upon repetitive stimulation at $5 \mathrm{mM}\left[\mathrm{K}^{+}\right]_{\mathrm{o}}\left(s s s^{\mathrm{P} 1} / s s s^{\mathrm{P} 1}\right.$, UAS$s s s ; 24 \mathrm{~B}-\mathrm{GAL} 4 /+=5.0 \pm 3.2 \%$ vs $s s s^{\mathrm{P} 1} ; 24 \mathrm{~B}-\mathrm{GAL} 4 /+=$ $39.3 \pm 3.9 \%, p<0.001)$ while not affecting the initial $I_{\mathrm{A}}$ magnitude (7.8 \pm 0.6 vs $7.4 \pm 0.8 \mathrm{nA} / \mathrm{nC}, p=0.72)$.

The finding that high $\left[\mathrm{K}^{+}\right]_{\mathrm{o}}$ partially antagonizes cumulative inactivation of $I_{\mathrm{A}}$ in $s s s^{\mathrm{P} 1}$ flies suggested that the shortened sleep phenotype of $s s s^{\mathrm{P} 1}$ flies may also be sensitive to $\left[\mathrm{K}^{+}\right]_{\mathrm{o}}$. As small molecules can be introduced effectively into live Drosophila to manipulate behavioral phenotypes (e.g., octopamine) (Monastirioti et al., 1996), we supplemented the fly food with up to 100 $\mathrm{mm} \mathrm{KCl}$ but found no rescue of sleep using the accepted Drosophila sleep-recording techniques (Pfeiffenberger et al., 2010).

\section{Discussion}

In our in vitro system, SSS increased the rate of Shaker channel activation without affecting the rate of deactivation. While SSS also increased the rate of decay of heterologously expressed wildtype Shaker currents at voltages $<30 \mathrm{mV}$, this may be an indirect effect of enhancing the rate of channel opening. As illustrated by kinetic modeling in Figure $5 A$, enhancing the rate of activation of inactivating channels not only hastens the time-to-peak, but also increases the rate of current decay. Thus, an increase in activation alone is sufficient to explain the in situ and in vitro effects of SSS on wild-type Shaker channels.

Consistent with previous reports (Wang et al., 2000; Wang and $\mathrm{Wu}, 2010$ ), we found that repetitive depolarization of $q \mathrm{vr} / \mathrm{sss}$ mutant larval muscles leads to a significant loss of Shaker current. The cumulatively inactivated component, recently named $I_{\mathrm{AS}}$ (Wang and $\mathrm{Wu}, 2010$ ), is eliminated by muscle-directed restoration of SSS in $s s^{\mathrm{P} 1}$ mutants, suggesting that SSS normally prevents the cumulative inactivation of Shaker currents in wild-type flies. In our in vitro studies, SSS did not affect recovery from either $\mathrm{N}$ - or C-type inactivation, but it decreased the rate of entry 
A

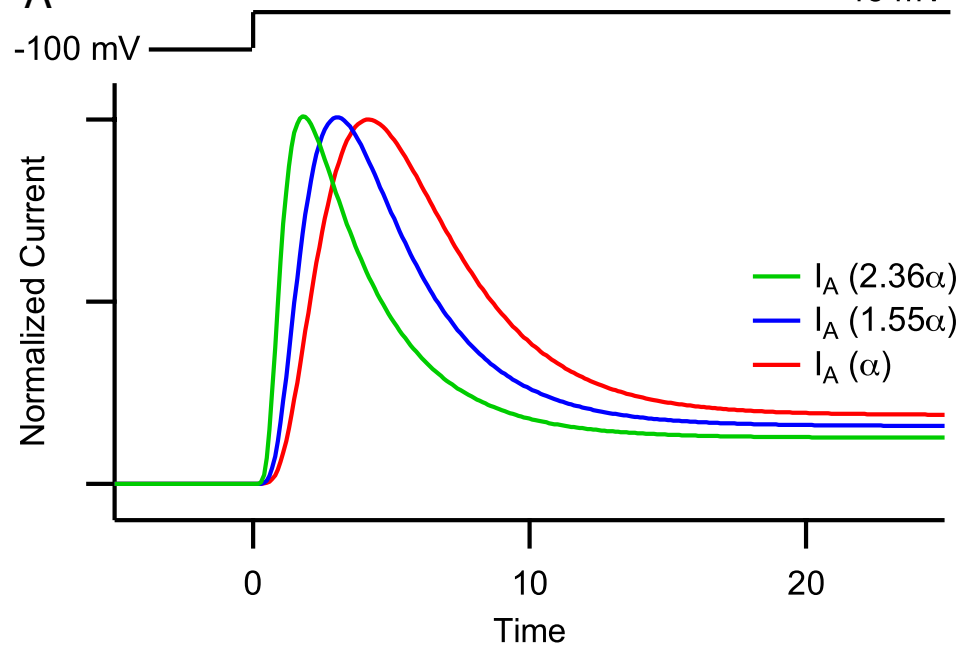

B

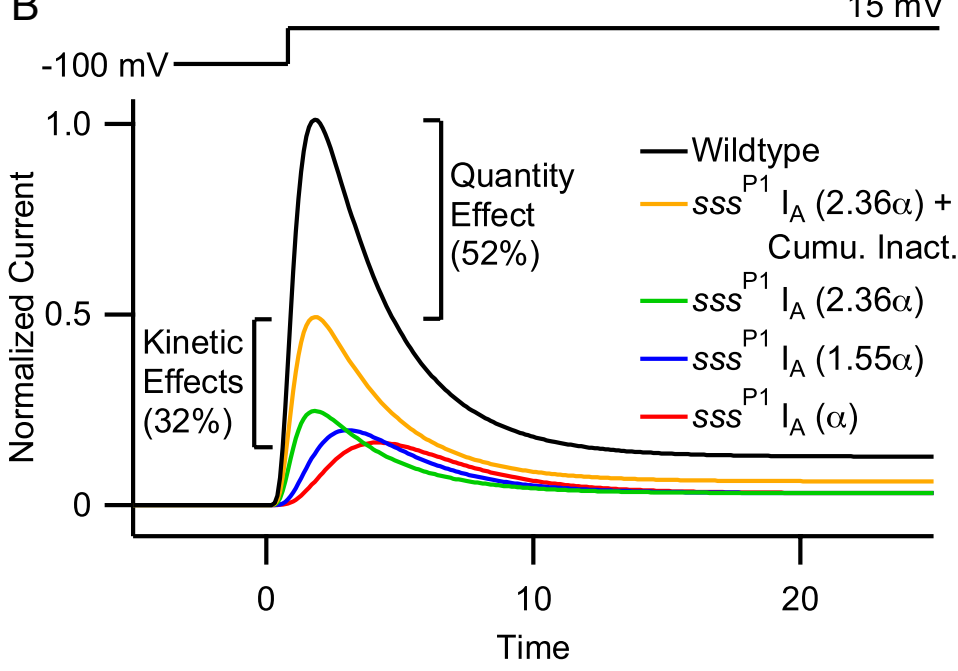

Figure 5. The SSS-dependent effects on Shaker kinetics comprise a significant proportion of $i$ situ I $A$ magnitude. $A$, Simulated ZHA traces in which an inactivation process has been restored, thus describing wild-type ShB currents. Enhancing the forward rate constant $\alpha$ decreases the time to peak and elicits a secondary increase in the rate of current decline (apparent $\tau_{\text {Decay }}=3.4,3.2$, and $2.8 \mathrm{~ms}$ at $\alpha, 1.55 \alpha$, and $2.36 \alpha$, respectively). These traces were normalized to peak current to illustrate the kinetic effect. $\boldsymbol{B}$, Simulated ZHA traces to depict the differential contributions of changes in Shaker kinetics and quantity to the overall $s s{ }^{\mathrm{P} 1} I_{\mathrm{A}}$ phenotype in situ. After repetitive depolarization, $s s{ }^{\mathrm{P} 1}$ mutants exhibit an approximate $84 \%$ decrease in $I_{\mathrm{A}}$ current $i n$ situ. We have simulated this loss using the ZHA model (black vs red). Relative to the $\operatorname{sss}^{\mathrm{P}}{ }^{\mathrm{P}} I_{\mathrm{A}}$ (red), the ZHA model predicts that the SSS-induced enhancement in activation kinetics may yield up to a $40 \%$ increase in Shaker current magnitude and a faster time-to-peak (blue and green). Furthermore, recovery of current lost to cumulative inactivation upon repeated stimulation nearly doubles the total $I_{\mathrm{A}}$ (orange vs green). Thus, the combined kinetic effects of $s s s^{P 1}$ make up an approximate $32 \%$ decrease in $I_{A}$ relative to wild-type. Presumably the remaining $52 \%$ of $I_{A}$ absent in $s s{ }^{P 1}$ flies is due to a decrease in Shaker quantity, consistent with the approximate $60 \%$ decrease in Shaker quantity observed by Western blot (Wu et al., 2010).

into the C-type inactivated state. SSS may also slow C-type inactivation of Shaker in Drosophila, as the cumulative inactivation phenotype observed in qvr/sss mutants could be caused by an absence of SSS-dependent resistance to C-type inactivation. This hypothesis is supported by the reduction of cumulative inactivation at supraphysiological concentrations of extracellular $\mathrm{K}^{+}$. However, direct quantitative comparisons between the in situ Shaker currents and the in vitro currents of ShB $\Delta \mathrm{N} T 449 \mathrm{~A}$ cannot be made, as the precise Shaker isoforms underlying the former are unknown, and the latter has a fundamentally different rate of C-type inactivation than wild-type channels. Similarly, testing the specific contribution of C-type inactivation to the $s s s^{\mathrm{P} 1}$ behavioral phenotypes may also be difficult. We were unable to rescue the $s s{ }^{\mathrm{P} 1}$ phenotype with a high $\mathrm{K}^{+}$diet but suspect that systemic $\mathrm{K}^{+}$concentration in Drosophila is tightly regulated, as it is in mammals.

Mutations in sss profoundly decrease Drosophila $I_{\mathrm{A}}$ magnitude (Wang et al., 2000; Wu et al., 2010); however, it is unclear whether the loss of Shaker current in $q v r / s s s$ mutants is caused by decreases in Shaker expression or changes in Shaker kinetics. Kinetic modeling based on in vitro experiments suggest that kinetic effects of SSS account for nearly $40 \%$ of the total loss in $I_{\mathrm{A}}$ magnitude exhibited by $s s s^{\mathrm{P} 1}$ mutants (Fig. $5 B$ ); the rest is likely due to a decrease in channel quantity. Interestingly, the cumulative inactivation of $I_{\mathrm{A}}$ comprises a large portion of the in situ current loss. Because the rescue of the cumulative inactivation phenotype nearly doubles the Shaker magnitude after repetitive depolarization, this kinetic rescue could account for the entirety of the previously reported partial rescue of total Shaker current magnitude (Wu et al., 2010). Therefore, in sss ${ }^{\mathrm{P1}}$ mutants, targeted expression of SSS completely rescues altered Shaker gating kinetics, cumulative inactivation, and slower $t_{\text {Peak }}$, as previously reported (Wu et al., 2010), while only minimally rescuing total Shaker current magnitude. Nevertheless, SSS expression fully rescues the sss ${ }^{\mathrm{P} 1}$ mutant sleep phenotype when driven broadly throughout the Drosophila brain as well as selectively in cholinergic neurons (Wu et al., 2010). Thus, the severity of the sleep phenotype in $s s{ }^{\mathrm{P} 1}$ mutants may better correlate with an alteration in Shaker current kinetics than magnitude. For example, SSS overexpression in wild-type flies reduces Shaker current in Drosophila muscles but does not induce a sleep phenotype, even when expressed using drivers that rescued the sleep phenotype in sss $^{\mathrm{P} 1}$ flies (Wu et al., 2010). Additionally, Drosophila bearing Shakernull alleles exhibit a less severe sleep phenotype than $s s s^{\mathrm{P} 1}$ mutants despite having no Shaker current (Cirelli et al., 2005; Koh et al., 2008). Because organisms can compensate for constitutive changes in ion channel quantity to preserve a neuron's overall excitability (MacLean et al., 2003; Lee et al., 2008), we speculate that static decreases in Shaker quantity are easier to compensate for than the dynamic, use-dependent loss of Shaker $\mathrm{K}^{+}$current seen in qvr/sss mutants. Future studies of in vivo central neurons in adult Drosophila (Crocker et al., 2010) may better elucidate the neurophysiological significance of the kinetic effects of SSS on Shaker.

The Shaker $\beta$-subunit Hyperkinetic (Hk) accelerates rate of Shaker activation (Chouinard et al., 1995) to a degree similar to that induced by SSS; however, Hk is an intracellular protein whereas SSS is tethered to the extracellular side of the membrane by a GPI-anchor. Although GPI-anchored auxiliary subunits have not yet been proposed for voltage-gated $\mathrm{K}^{+}$channels, a recent study found that the voltage-gated $\mathrm{Ca}^{2+}$ channel auxiliary subunit $\alpha_{2} \delta$ is indeed GPI-anchored (A. Davies et al., 2010). Just 
as adequate $\mathrm{Ca}^{2+}$ channel expression depends on $\alpha_{2} \delta$, a deficiency of SSS leads to decreases in Shaker protein levels in vivo (Wu et al., 2010). Additionally, $\alpha_{2} \delta$-containing $\mathrm{Ca}^{2+}$ channels concentrate in lipid rafts, much like SSS and Shaker. Furthermore, the SSS-induced increase in activation of heterologously expressed Shaker channels was sensitive to cholesterol depletion, similar to $\mathrm{K}^{+}$currents in ex vivo neurons from the Drosophila mushroom bodies (Gasque et al., 2005), raising the possibility that the mechanism by which SSS interacts with Shaker involves lipid rafts. Thus, GPI-anchored proteins like SSS and $\alpha_{2} \delta$ may be critical regulators of ion channel function and quantity at the cell surface.

SSS enhances gating charge movement, but the mechanism and stoichiometry by which SSS affects the charge-bearing S4 transmembrane segment remain unknown. Because SSS and Shaker coimmunoprecipitate when coexpressed in a heterologous system (Wu et al., 2010), one potential mechanism is a direct protein-protein interaction between SSS and the extracellular loops of Shaker. The S1-S2 linker is a promising candidate, as a threonine to isoleucine substitution in this loop $\left(S h^{\mathrm{mns}}\right)$ also induces a sleep phenotype; however, the functional consequences of that allele have not yet been thoroughly characterized (Cirelli et al., 2005).

Increasing the forward rate constant $\alpha$ in the two-step model of Shaker activation (Zagotta et al., 1994a) was sufficient to reproduce the effects of SSS on the activation of ionic currents and the time course of gating charge movement. A recent structurefunction study of Shaker gating by Tao et al. (2010) suggests that activation requires at least four transitions between five discrete states of the $S 4$ voltage sensor. The forward rate constant $\alpha$ in the ZHA model may correspond to the initial transitions of the voltage sensor, and thus SSS may act upon the early intermediate states of S4, including destabilizing the resting state when the first charge of S4 is located below the S2 phenylalanine cap.

There are also potential mechanisms for the SSS-dependent modulation of activation and C-type inactivation aside from a direct SSS-Sh interaction. For instance, SSS may indirectly act upon Shaker through another protein, as even in an in vitro system like HEK cells, native proteins found in lipid rafts (e.g., caveolin) can modulate $\mathrm{K}^{+}$channels (L. M. Davies et al., 2010). Another potential effect of SSS may be to increase local extracellular $\mathrm{K}^{+}$accumulation upon channel opening and $\mathrm{K}^{+}$efflux, therefore indirectly slowing C-type inactivation. SSS may even affect channel kinetics by altering interactions between surrounding lipids and Shaker channels, an important consideration given that membrane phosopholipids play critical roles in ion channel function (Xu et al., 2008). A lipid-channel interaction mediated by SSS is consistent with our finding that the sigmoidicity of macroscopic current activation was unaltered by SSS, regardless of the degree of acceleration that SSS induced. Had the interaction between SSS and Shaker followed a strict stoichiometry in which individual subunits within a tetramer were selectively enhanced, then it is unlikely that activation sigmoidicity could have been preserved over the range of activation speeds observed. Because sigmoidicity is preserved over the entire range of activation speeds, we suspect that every subunit of each channel in a patch is equally affected by the variable expression levels of SSS. The effect may be unique to Shaker, as human Slo1 activation kinetics are not affected by SSS expression despite also trafficking to lipid rafts (Weaver et al., 2007), suggesting that either Shaker is particularly sensitive to lipid membrane composition or that cholesterol plays a permissive role in the effects of
SSS on Shaker kinetics. Nevertheless, we cannot entirely exclude effects of SSS on channels other than Shaker.

In summary, SSS regulates the kinetics of Shaker channels on timescales ranging from milliseconds to hundreds of milliseconds. These kinetic effects, in addition to the SSS-dependent effects on Shaker expression and subcellular localization (Wu et al., 2010), suggest that SSS is an important regulator of Shaker and that the modulation of Shaker $\mathrm{K}^{+}$conductance is a critical component of the sleep behavior in Drosophila.

\section{References}

Borbély AA, Achermann P (1999) Sleep homeostasis and models of sleep regulation. J Biol Rhythms 14:557-568.

Choi KL, Aldrich RW, Yellen G (1991) Tetraethylammonium blockade distinguishes two inactivation mechanisms in voltage-activated $\mathrm{K}^{+}$channels. Proc Natl Acad Sci U S A 88:5092-5095.

Chouinard SW, Wilson GF, Schlimgen AK, Ganetzky B (1995) A potassium channel beta subunit related to the aldo-keto reductase superfamily is encoded by the Drosophila hyperkinetic locus. Proc Natl Acad Sci U S A 92:6763-6767.

Cirelli C, Bushey D, Hill S, Huber R, Kreber R, Ganetzky B, Tononi G (2005) Reduced sleep in Drosophila Shaker mutants. Nature 434:1087-1092.

Crocker A, Shahidullah M, Levitan IB, Sehgal A (2010) Identification of a neural circuit that underlies the effects of octopamine on sleep:wake behavior. Neuron 65:670-681.

Davies A, Kadurin I, Alvarez-Laviada A, Douglas L, Nieto-Rostro M, Bauer CS, Pratt WS, Dolphin AC (2010) The $\alpha_{2} \delta$ subunits of voltage-gated calcium channels form GPI-anchored proteins, a posttranslational modification essential for function. Proc Natl Acad Sci U S A 107:1654-1659.

Davies LM, Purves GI, Barrett-Jolley R, Dart C (2010) Interaction with caveolin-1 modulates vascular ATP-sensitive potassium $\left(\mathrm{K}_{\mathrm{ATP}}\right)$ channel activity. J Physiol 588:3255-3266.

Demo SD, Yellen G (1991) The inactivation gate of the Shaker $\mathrm{K}^{+}$channel behaves like an open-channel blocker. Neuron 7:743-753.

Feng Y, Ueda A, Wu CF (2004) A modified minimal hemolymph-like solution, HL3.1, for physiological recordings at the neuromuscular junctions of normal and mutant Drosophila larvae. J Neurogenet 18:377-402.

Gasque G, Labarca P, Darszon A (2005) Cholesterol-depleting compounds modulate $\mathrm{K}^{+}$-currents in Drosophila Kenyon cells. FEBS Lett 579:5129-5134.

Gilestro GF, Cirelli C (2009) pySolo: a complete suite for sleep analysis in Drosophila. Bioinformatics 25:1466-1467.

Hoshi T, Zagotta WN, Aldrich RW (1990) Biophysical and molecular mechanisms of Shaker potassium channel inactivation. Science 250:533-538.

Hoshi T, Zagotta WN, Aldrich RW (1991) Two types of inactivation in Shaker $\mathrm{K}^{+}$channels: effects of alterations in the carboxy-terminal region. Neuron 7:547-556.

Hoshi T, Zagotta WN, Aldrich RW (1994) Shaker potassium channel gating. I. Transitions near the open state. J Gen Physiol 103:249-278.

Koh K, Joiner WJ, Wu MN, Yue Z, Smith CJ, Sehgal A (2008) Identification of SLEEPLESS, a sleep-promoting factor. Science 321:372-376.

Lee J, Ueda A, Wu CF (2008) Pre- and post-synaptic mechanisms of synaptic strength homeostasis revealed by slowpoke and shaker $\mathrm{K}^{+}$channel mutations in Drosophila. Neuroscience 154:1283-1296.

López-Barneo J, Hoshi T, Heinemann SH, Aldrich RW (1993) Effects of external cations and mutations in the pore region on C-type inactivation of Shaker potassium channels. Receptors Channels 1:61-71.

MacLean JN, Zhang Y, Johnson BR, Harris-Warrick RM (2003) Activityindependent homeostasis in rhythmically active neurons. Neuron 37:109-120.

Martens JR, Sakamoto N, Sullivan SA, Grobaski TD, Tamkun MM (2001) Isoform-specific localization of voltage-gated $\mathrm{K}^{+}$channels to distinct lipid raft populations: targeting of Kv1.5 to caveolae. J Biol Chem 276:8409-8414.

Miwa JM, Ibanez-Tallon I, Crabtree GW, Sánchez R, Sali A, Role LW, Heintz N (1999) Lynxl, an endogenous toxin-like modulator of nicotinic acetylcholine receptors in the mammalian CNS. Neuron 23:105-114.

Monastirioti M, Linn CE Jr, White K (1996) Characterization of Drosophila tyramine beta-hydroxylase gene and isolation of mutant flies lacking octopamine. J Neurosci 16:3900-3911. 
Perozo E, MacKinnon R, Bezanilla F, Stefani E (1993) Gating currents from a nonconducting mutant reveal open-closed conformations in Shaker $\mathrm{K}^{+}$ channels. Neuron 11:353-358.

Pfeiffenberger C, Lear BC, Keegan KP, Allada R (2010) Processing sleep data created with the Drosophila activity monitoring (DAM) system. Cold Spring Harb Protoc 2010:pdb.prot5520.

Rietveld A, Neutz S, Simons K, Eaton S (1999) Association of sterol- and glycosylphosphatidylinositol-linked proteins with Drosophila raft lipid microdomains. J Biol Chem 274:12049-12054.

Tao X, Lee A, Limapichat W, Dougherty DA, MacKinnon R (2010) A gating charge transfer center in voltage sensors. Science 328:67-73.

Van Dongen HP, Maislin G, Mullington JM, Dinges DF (2003) The cumulative cost of additional wakefulness: dose-response effects on neurobehavioral functions and sleep physiology from chronic sleep restriction and total sleep deprivation. Sleep 26:117-126.

Wang JW, Wu CF (2010) Modulation of the frequency response of Shaker potassium channels by the quiver peptide suggesting a novel extracellular interaction mechanism. J Neurogenet 24:67-74.

Wang JW, Humphreys JM, Phillips JP, Hilliker AJ, Wu CF (2000) A novel leg-shaking Drosophila mutant defective in a voltage-gated $\mathrm{K}^{+}$current and hypersensitive to reactive oxygen species. J Neurosci 20:5958-5964.

Weaver AK, Olsen ML, McFerrin MB, Sontheimer H (2007) BK channels are linked to inositol 1,4,5-triphosphate receptors via lipid rafts: a novel mechanism for coupling $\left[\mathrm{Ca}^{2+}\right]_{\mathrm{i}}$ to ion channel activation. J Biol Chem 282:31558-31568.
Wu CF, Haugland FN (1985) Voltage clamp analysis of membrane currents in larval muscle fibers of Drosophila: alteration of potassium currents in Shaker mutants. J Neurosci 5:2626-2640.

Wu MN, Koh K, Yue Z, Joiner WJ, Sehgal A (2008) A genetic screen for sleep and circadian mutants reveals mechanisms underlying regulation of sleep in Drosophila. Sleep 31:465-472.

Wu MN, Joiner WJ, Dean T, Yue Z, Smith CJ, Chen D, Hoshi T, Sehgal A, Koh K (2010) SLEEPLESS, a Ly-6/neurotoxin family member, regulates the levels, localization and activity of Shaker. Nat Neurosci 13:69-75.

Xu Y, Ramu Y, Lu Z (2008) Removal of phospho-head groups of membrane lipids immobilizes voltage sensors of $\mathrm{K}^{+}$channels. Nature 451:826-829.

Yancey PG, Rodrigueza WV, Kilsdonk EP, Stoudt GW, Johnson WJ, Phillips MC, Rothblat GH (1996) Cellular cholesterol efflux mediated by cyclodextrins: demonstration of kinetic pools and mechanism of efflux. J Biol Chem 271:16026-16034

Zagotta WN, Hoshi T, Aldrich RW (1989) Gating of single Shaker potassium channels in Drosophila muscle and in Xenopus oocytes injected with Shaker mRNA. Proc Natl Acad Sci U S A 86:7243-7247.

Zagotta WN, Hoshi T, Aldrich RW (1994a) Shaker potassium channel gating. III. Evaluation of kinetic models for activation. J Gen Physiol 103:321-362.

Zagotta WN, Hoshi T, Dittman J, Aldrich RW (1994b) Shaker potassium channel gating. II. Transitions in the activation pathway. J Gen Physiol 103:279-319. 